\title{
Biobanking of human gut organoids for translational research
}

\author{
Francesca Perrone $e^{1}$ and Matthias Zilbauer (iD) $1,2,3$
}

\begin{abstract}
The development of human organoid culture models has led to unprecedented opportunities to generate selforganizing, three-dimensional miniature organs that closely mimic in vivo conditions. The ability to expand, culture, and bank such organoids now provide researchers with the opportunity to generate next-generation living biobanks, which will substantially contribute to translational research in a wide range of areas, including drug discovery and testing, regenerative medicine as well as the development of a personalized treatment approach. However, compared to traditional tissue repositories, the generation of a living organoid biobank requires a much higher level of coordination, additional resources, and scientific expertise. In this short review, we discuss the opportunities and challenges associated with the generation of a living organoid biobank. Focusing on human intestinal organoids, we highlight some of the key aspects that need to be considered and provide an outlook for future development in this exciting field.
\end{abstract}

\section{Introduction}

A vast amount of human tissue is being collected for routine clinical purposes on a daily basis, and often, only a fraction of the available material is required. It has long been recognized that the prospective storage of such excess tissue in the form of biobanks or tissue banks provides extensive opportunities for future research studies $^{1-6}$. Indeed, many ground-breaking discoveries in both basic and translational biomedical research have benefitted from the analyses of human tissue stored in large biobanks. Importantly, the value of such tissue banks is directly associated with the quality of their organization, which includes the detailed documentation of patient information as well as sample processing and storage. Major progress has been made in this field, leading to the development of large, highly sophisticated biobanks ${ }^{7-10}$. However, a major limitation of stored tissue is the inability

Correspondence: Matthias Zilbauer (mz304@cam.ac.uk)

'Department of Paediatrics, University of Cambridge, Addenbrooke's Hospital, Cambridge, UK

${ }^{2}$ Department of Paediatric Gastroenterology, Hepatology and Nutrition,

Cambridge University Hospitals, Addenbrooke's, Cambridge, UK

Full list of author information is available at the end of the article to perform functional experiments, which are required for many areas, particularly in translational research. The development of human organoid culture models has provided researchers with unprecedented opportunities to generate miniature organs that closely mimic in vivo anatomy and pathophysiology and retain patient-specific characteristics $^{11}$. To date, organoids have been successfully generated from a wide range of human tissues, including the entire intestinal tract ${ }^{12-17}$. Indeed, since the development of the first organoid culture model from the mouse small intestine approximately one decade ago ${ }^{18}$, substantial progress has been made in this area ${ }^{19-21}$. Although the majority of topics discussed in this review will equally apply to any organoid biobank, we will focus on the human intestine based on our own experience in this field.

Intestinal organoids are defined as self-organizing threedimensional structures that closely mimic the in vivo situation. In principle, they can be generated either from pluripotent stem cells (e.g., induced pluripotent stem cells (iPSCs) or embryonic stem cells (ESCs)) or adult stem cells $^{22-24}$. The latter is located in the crypts of the intestinal epithelium and, upon provision of the 
appropriate scaffold and culture conditions that mimic the stem cell niche, will self-organize into mature intestinal epithelial organoids (IEOs), which contain all epithelial cell subsets structured in a crypt villus axis. To date, IEOs have been successfully generated from all gut segments of the human intestinal tract, and several studies have shown that organoids display gut segment-specific features in vitro ${ }^{25-27}$. Furthermore, increasing evidence suggests that patient-derived IEOs retain donor-specific properties such as age, gender, and disease-associated differences $^{9,28,29}$. As a result, IEOs provide scientists with unprecedented opportunities to use these models as highly versatile translational research tools. Areas of clinical relevance include but are by no means limited to the development of novel therapeutics, testing of existing drugs with an aim to personalize treatment based on responses as well as the use in regenerative medicine (Fig. 1a) ${ }^{11,30-32}$.

Importantly, IEOs can be expanded in vitro over prolonged time periods and frozen stocks can be generated at any time during this process. This phenomenon allows the generation of living biobanks and biorepositories that can be shared with researchers across the globe and in particular those who do not have access to human tissue samples (Fig. 1b). However, despite the extraordinary progress in the field of human IEOs over the last decade, in regard to translating science into patient care, additional caution and stringent quality control procedures are critical first steps. In the following, we will briefly summarize examples of existing human organoid biobanks and highlight considerations for generating and distributing IEOs as part of a biobank or biorepository. Finally, we will provide an outlook on future developments and key strategic goals for the field. We will primarily focus on mucosa-derived IEOs, as our group has extensive expertise in this field. Nevertheless, most aspects we discuss will also apply to iPSC-derived gut organoids, and we therefore will not distinguish between them unless otherwise specified.

\section{Existing human intestinal organoid biobanks}

The major value of generating organoid biobanks for translational research purposes has long been recognized and is reflected in the rapidly increasing number of studies reporting on the successful establishment of large patient-derived, living biobanks. Interestingly, the vast majority of such sample collections have been derived from patients diagnosed with various malignancies, including those affecting the gastrointestinal tract. Examples include colorectal cancer, metastatic colorectal and esophageal cancer, gastric cancer, pancreatic cancer, and advanced rectal cancer (summarized in Table 1$)^{9,28,29,33-38}$. In all of these cases, the generation of patient-derived biobanks included a detailed description of clinical phenotype and patient records, characteristics of samples used to generate organoids (such as macroscopic and/or histological appearance), as well as molecular profiling of both the tissue of origin and/or the resulting organoid cultures. Furthermore, most studies also provide evidence for generated organoids to recapitulate patient and disease-specific pathophysiological processes, thereby validating them as translational research tools ${ }^{9,28,29}$. Importantly, several studies have highlighted the ability of generated organoids to personalize treatment by demonstrating a patient-specific response to existing treatments ${ }^{34,35}$.

In contrast to reports on malignancies, only a limited number of studies have been able to demonstrate the value of living organoid biobanks for other GI-related conditions. This phenomenon is in part due to the presence of genetic alterations in malignant tissue which are faithfully retained in organoid cultures and impact cellular function. Indeed, cystic fibrosis (CF) represents another example of a genetic, nonmalignant condition for which the value of patient-derived organoids as translational tools to stratify treatment has been demonstrated. CF is caused by various mutations in the CF transmembrane conductance regulator (CFTR) gene ${ }^{39}$. Human IEOs derived from rectal biopsies of CF patients have shown a strong correlation with in vivo drug responses by using a forskolin-induced swelling assay in vitro ${ }^{40}$. Based on these discoveries, a living organoid biobank was generated representing over 600 patient-derived organoid cultures ${ }^{36}$. Notably, all generated organoid lines were shown to retain patient-specific mutations in the CFTR gene, some of which could be corrected using SpCas9-induced DNA template recombination or SpCas9-adenine base editing $^{36,41}$. The latter fact further highlights the use of genome editing to further enhance the value of organoid cultures as translational research tools.

One of the most extensive organoid biobanks was established by the The Hubrecht Institute, the University Medical Center Utrecht, and the Royal Netherlands Academy of Arts and Sciences (KNAW) as part of Hubrecht Organoid Technology (HUB, https:// huborganoids.nl/). The collection contains more than 1000 organoid lines generated from different organs and diseases, including breast, colon, head, neck, intestine, liver, lung, ovarian, and pancreatic tumors, as well as a large number of intestinal organoids derived from CF patients. Genetic and histological data of all lines are available along with detailed information about the sensitivity to specific drugs for treatment strategies in the case of CF patient-derived organoids.

In addition to academic institutions, organoid biobanks have been established by commercial entities such as Sigma-Aldrich, the American Type Culture Collection (ATCC, (www.attc.org)), Cellesce (https://cellesce.com) 
a)

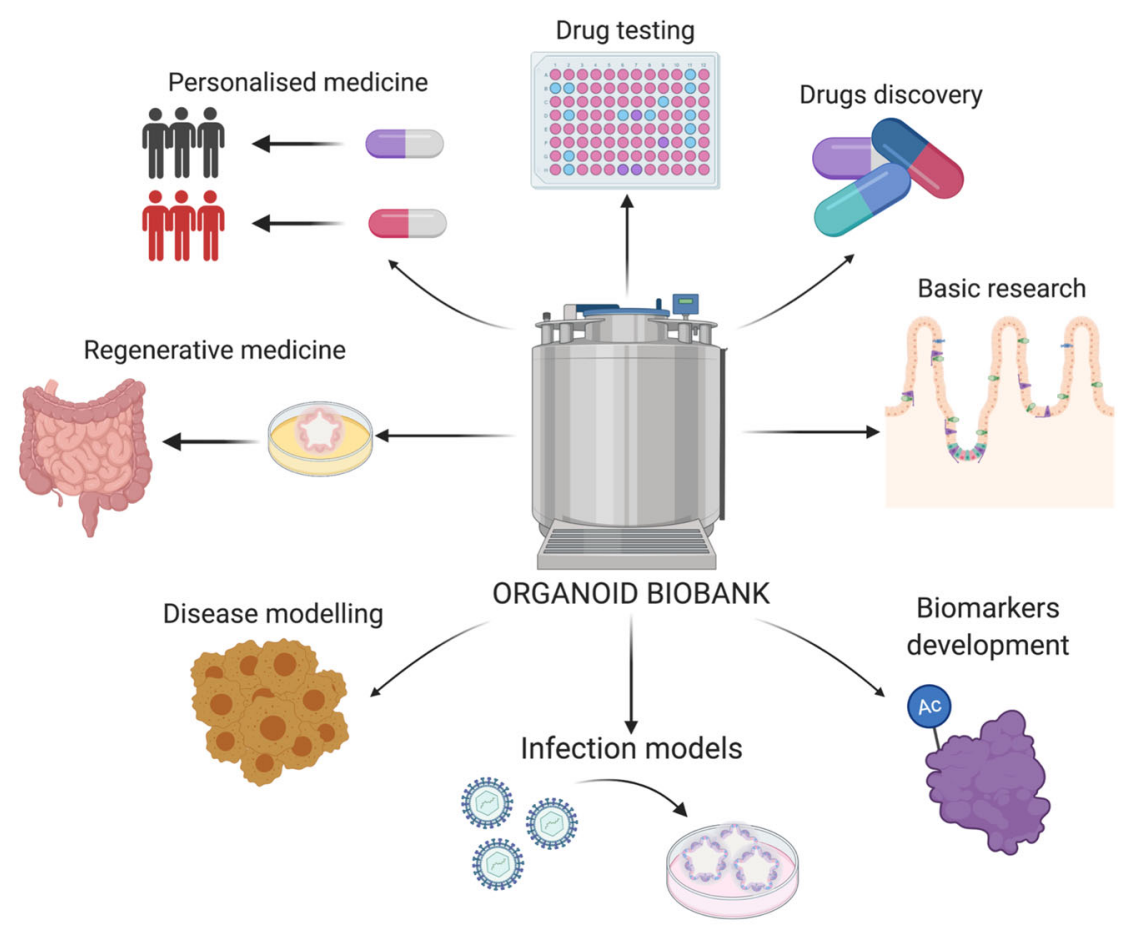

b)

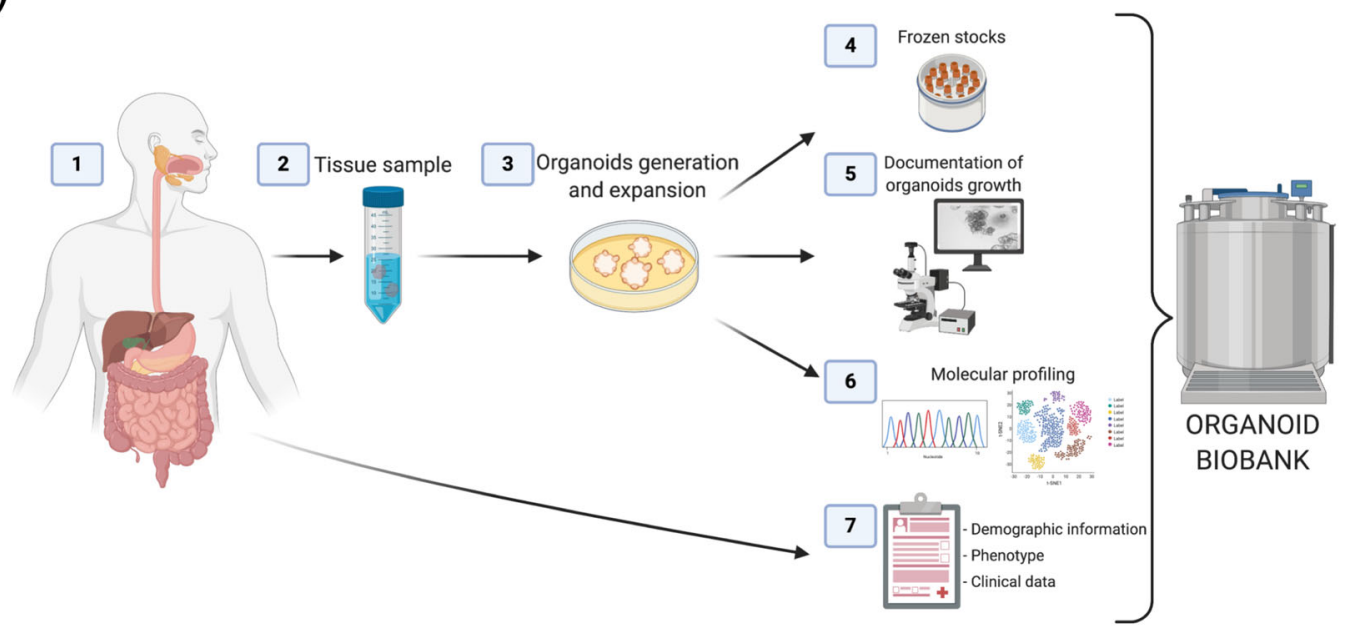

Fig. 1 Establishment of an organoid biobank and its applications. a Applications for next-generation organoid biobanks in translational research. b Basic workflow for the generation of an organoid biobank. Key areas requiring careful consideration and detailed documentation include (1) donor information, (2) tissue of origin, (3) culture methodology and passage number, (4) generation of frozen stocks, (5) documentation of organoid growth and morphology, (6) molecular profiling of organoids and/or primary tissue, and (7) relevant clinical information. All images were created with BioRender.com.

and DefiniGEM (https://www.definigen.com/products/ intestinal/organoid/). These collections offer a wide range of human organoids derived from either iPSCs or primary tissues (Table 1) covering both healthy donors and patients with established diseases. Detailed information on both generated organoid cultures and donors is also available and includes molecular profiling, the results of drug screening, and the presence of specific genetic mutations in some instances. A proportion of organoid lines available via these biobanks were generated from iPSCs, further extending the experimental repertoire and potential applications of cultures available to researchers. 


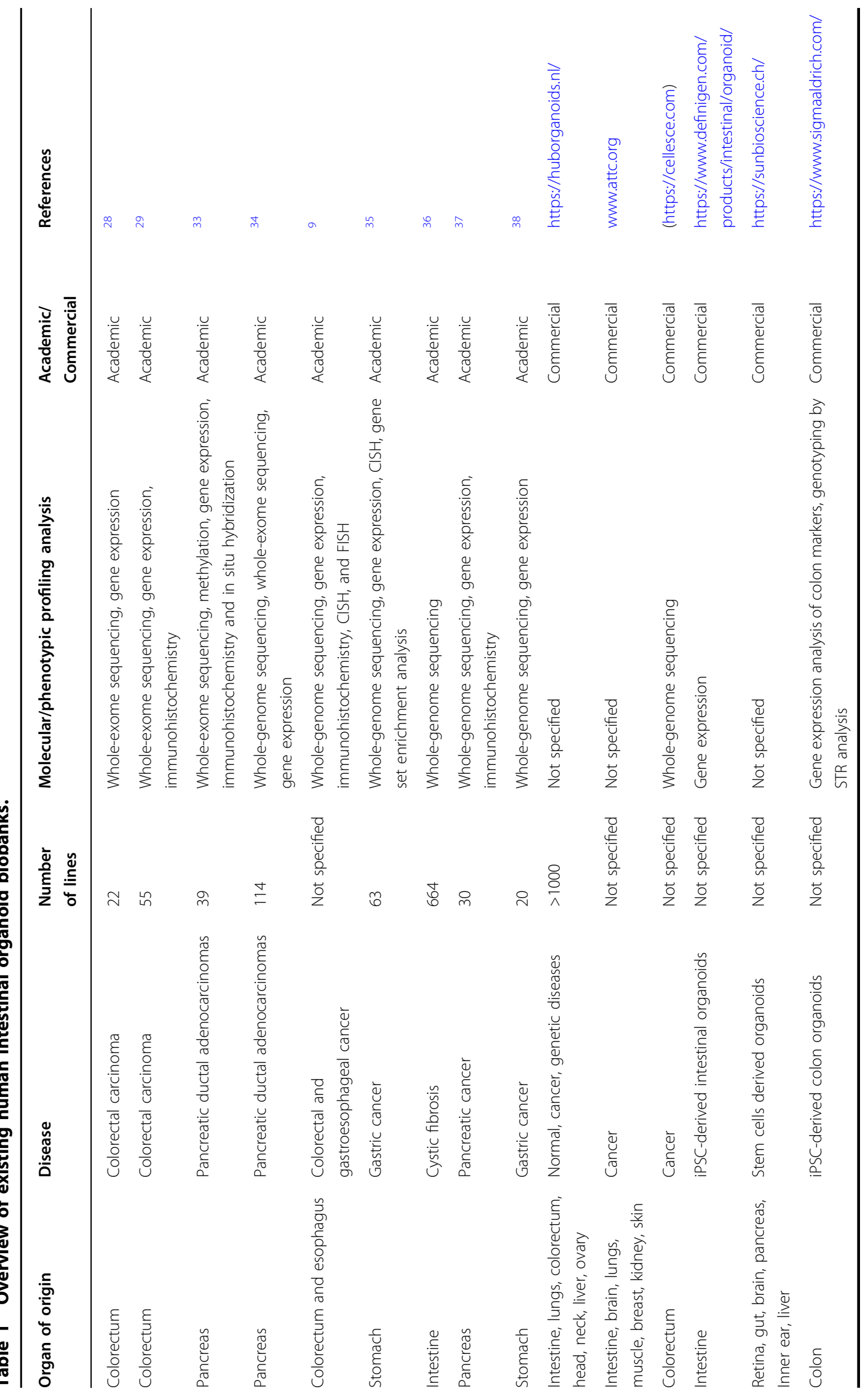


In summary, the number of organoid biobanks and repositories has been increasing in recent years, with a large proportion of disease-related collections focused on malignant and/or genetically well-defined conditions.

\section{Important features of organoid biobanks}

Despite major progress made in this field and the high quality of human organoid biobanks established to date, several areas require consideration to further improve the value of living organoid biobanks in the future. In the following, we will summarize some of the key aspects that are critical for the generation of a living organoid biobank to enhance their value for translational research.

\section{Patient and sample details}

One of the most important advantages of patient-derived organoids is the fact that these cultures retain the molecular as well as functional characteristics of their donors ${ }^{26,28}$. Hence, providing detailed donor information directly correlates with the utility of generated organoids. In addition to basic characteristics such as gender, age, ethnic background, and potential disease, additional information such as medication, diet, family history of any medical conditions, or the presence of gastrointestinal symptoms are all highly relevant to a wide range of possible research questions. Furthermore, prospective documentation of disease outcome and treatment history, including response to individual treatments, will allow the correlation of organoid-derived molecular signatures or potential functional differences with clinical phenotype. In other words, the documentation of patient details forms the basis for the use of organoids as tools to develop personalized treatment approaches and/or clinical biomarkers (Fig. 1b).

Similarly, the type of tissue samples used to generate IEOs is also critically important. The tissue samples used vary from mucosal biopsies (obtained during routine clinically indicated endoscopy) and surgical resection material to deceased tissue donation. Each of these tissue types may impact the cellular function of generated organoids even if samples were obtained from the same organ/gut segment. In addition, the time from obtaining tissue to initiating organoid cultures, including specification about possible tissue storage, may also impact downstream analyses and should therefore be recorded. Finally, the macroscopic and histological appearance of the intestinal mucosa from which the tissue sample was taken provides highly valuable information on the in vivo microenvironment at the time of sampling and is therefore relevant for the interpretation of experimental results obtained from generated organoids. Providing representative histological images (e.g., hematoxylin and eosin stains) of the original tissue used to generate organoids further enhances the value of the generated lines for future applications.
We would like to highlight the fact that there is currently a lack of published evidence to support the possible impact of the factors mentioned above on generated organoids. However, based on our experience and unpublished data, we believe that these factors are indeed critically important, and unfortunately, many existing studies lack the required information. Hence, prospective, detailed documentation will help to address these important issues in the future and aid the interpretation of generated data as part of published work.

In summary, the value and future use of an organoid biobank for basic and translational research studies critically depend on the information provided on donor and tissue samples used to generate cultures. Despite the major complexities and additional work required to record such details, the increase in value will no doubt outweigh the invested resources.

\section{Culture conditions and documentation of growth}

The initiation and long-term culture of human IEOs were first described by Sato et al. ${ }^{15}$ just over one decade ago. Since then, major progress has been made with regard to optimizing existing protocols. Although the basic culture conditions used are largely comparable between studies and research groups, even subtle variations could lead to substantial differences in organoid phenotype, growth or any downstream analyses performed. One example of such variation is the source of Wnt3a, which is a key component of human IEOs culture medium $^{42,43}$. Although Wnt3a is commercially available as a recombinant protein, its use in the culture of human gut organoids has been reported to be insufficient, as it results in reduced organoids growth ${ }^{44}$. As a result, many studies report the use of conditioned medium-producing cell lines as a source of Wnt3a ${ }^{45,46}$. However, although this approach appears to be superior to the use of recombinant protein, based on our experience, there is substantial variation in batches of conditioned medium, which may impact on organoid growth and/or molecular processes. More recently, a new next-generation surrogate Wnt ligand able to bind both the Wnt receptor (Frizzled) and coreceptors (Lrp5/6) was developed and shown to support the long-term growth of human IEOs ${ }^{47}$. The use of this next-generation recombinant Wnt surrogate will likely improve the reproducibility of organoid work as information on specific concentrations used can be provided. Another example of possible variation in organoid culture comes with the use of different scaffolding reagents to support culture growth. Matrigel, derived from mouse sarcoma, is among the most frequently used extracellular matrix scaffolds and has been shown to adequately support human gut organoid growth and 3D self-organization in many studies ${ }^{48,49}$. However, the composition of Matrigel remains ill-defined and 
appears to display substantial batch variation, which could impact on reproducibility and comparability of stu$\operatorname{dies}^{50,51}$. Alternative options include animal-derived gel matrixes such as collagen type I and the more recently developed synthetic scaffolds using artificial hydrogels such as polyethylene glycol (PEG), which showed comparable results to those of Matrigel in supporting organoids growth ${ }^{52,53}$. The latter benefits from a clearly defined composition and adjustable mechanical properties, which may reduce the risk of technical variation among studies ${ }^{54,55}$. These examples highlight the need for organoid repositories to provide detailed documentation of culture conditions used by paying particular attention to detail, as minor changes can lead to substantial differences in the results generated.

Regardless of the culture conditions used, documenting organoid growth and structure is critically important and highly valuable. This process can be performed using light microscopy at key stages, including budding stages following the first initiation of cultures, immediately after subculturing, and before frozen stocks are being generated. Information on how many organoids are frozen is also helpful for downstream users and should be considered as standard practice. Similarly, information is routinely provided when sharing cell lines, such as details on regular testing of tissue culture medium for contaminants ${ }^{56,57}$.

The number of passages and culture duration are also critically important and should therefore be recorded. Although human IEOs can be kept in culture for months, even years, the potential impact on cellular phenotype remains largely unknown. Indeed, it is highly likely that artificial culture conditions and the lack of certain external stimuli present in vivo (e.g., cell-cell signaling, microbiota, exposure to nutrients, etc.) may impact epithelial cell function and/or alter molecular profiles. Hence, the generation of robust evidence on the impact of culture duration on human organoids with regard to phenotypic changes (both transient and/or permanent) should be considered a priority in the field. Moreover, documentation of culture duration and passage number must form an essential part of the information provided (Fig. 1b).

\section{Cryopreservation of tissue for organoid generation}

The ability to generate organoids from cryopreserved human tissue has substantially increased the value of samples obtained both for routine clinical care and for specific research settings. A number of protocols have been published that allow cryopreservation of tissue samples followed by successful establishment of organoids even after long-term storage ${ }^{58-60}$. In principle, these protocols require tissue samples to be transferred to freezing medium followed by a gradual reduction in temperature down to $-80^{\circ} \mathrm{C}$ and transfer to liquid nitrogen storage ${ }^{59,61}$. Success rates for the generation of organoids from frozen tissue are likely to be lower than those for fresh tissue; however, based on our own experience, up to $80 \%$ of adequately cryopreserved gut biopsy samples will give rise to viable organoids lines ${ }^{59,62}$. Importantly, these methods will further broaden the application of human organoid technologies and the opportunity for the generation of large living biobanks, as samples can be obtained from patients treated at smaller hospitals that do not have the required research infrastructure to initiate organoid cultures themselves.

However, although based on our experience, organoids generated from frozen tissue samples appear to display the same growth characteristics and morphology as cultures derived from fresh tissue, a freeze-thaw cycle and long-term storage could impact on the cellular function of the generated organoid cultures. Further studies are needed to address this important issue. Thus, detailed information on freezing methods and storage duration should be routinely recorded.

\section{Molecular profiling of human IEOs}

In addition to providing detailed information on donors, tissues, and organoid culture, generating molecular profiles of stored IEOs and/or the primary tissue used to generate these cultures can vastly increase their future value for translational research studies (Fig. 1b). As outlined above, numerous studies have demonstrated the application of organoids in translational research studies by linking organoid function to molecular profiles, mainly genetic profiles (Table 1$)^{34,63-65}$. However, in contrast to the use of genotype to subclassify organoids and stratify possible treatments, other molecular profiles are more likely to change during in vitro culture, making it more challenging to identify a potential correlation with disease, phenotype or response to treatment. For example, transcriptional profiles generated from human IEOs will vastly vary according to a number of factors, such as culture duration following initiation or splitting, the level of differentiation (i.e., profiled in maintenance medium versus in vitro differentiation) in culture medium, and scaffolds and growth factors used. Other molecular signatures/ mechanisms, such as DNA methylation, might also be useful, as they have been shown to be highly stable in culture and retain both gut segment, age, and diseasespecific alterations in human IEOs ${ }^{25,66}$. Future studies will be able to elucidate these issues further and help to streamline the molecular profiling of organoid biobanks in the future. Currently, the provision of genotypes derived from the original tissue used to generate organoids will substantially increase their future use.

\section{Ethical considerations}

Although next-generation living organoid biobanks have substantial potential and provide unprecedented 
opportunities for future translational research, they raise numerous ethical issues beyond those that apply to sharing primary human tissue samples. Developments in this important area are ongoing, and due to the complexity of the subject, providing further details is beyond the scope of this review article. However, we refer the interested reader to a number of excellent articles on this topic which highlight some of the key issues including the difficulties in balancing maximizing the benefit of organoid culture technology by making them widely available and providing detailed accompanying information whilst at the same time protecting donor identity ${ }^{6,67-71}$. While close collaboration with pharmaceutical companies forms a vital step in the process of translating any academic discoveries from the bench to the bedside, the prospect of profit-making from human tissue donated for academic research is ethically contentious and likely to raise concern among the wider public ${ }^{72}$.

These are only some of the major ethical issues that require urgent and careful attention to ensure the timely development of sound guidelines for scientists, clinicians, and other stakeholders.

\section{Future directions and conclusions}

The storage and subsequent sharing of human organoid cultures as part of next-generation biobanks have the potential to substantially increase their value and provide major opportunities for basic and translational research. However, the complexities involved in both generating and culturing human organoids as well as storing not only the tissue but also relevant information provide significant additional challenges. Furthermore, given that organoid models are being rapidly developed and improved, validation and quality control studies are urgently needed to inform best practice and support the development of widely accepted protocols. Key areas include the potential impact of long-term in vitro culturing on molecular signatures and cellular function as well as more detailed validation studies aiming to determine the degree to which organoids retain diseasespecific features in culture. The potential impact of longterm storage and/or freeze-thaw cycles as fundamental aspects of biobanking must be further explored. Finally, the establishment of clear ethical guidelines that cover areas such as commercialization, patient consent, and the use of organoids for future, non-specified research are needed to ensure the will and privacy of donors are protected.

In conclusion, despite major challenges associated with the generation of next-generation living organoid biobanks, their future use in translational biomedical research is likely to contribute to many groundbreaking discoveries. A collaborative and well-organized effort from the scientific community is required to ensure timely developments in this exciting area.

\section{Acknowledgements}

This work was supported by an MRC New Investigator Research Grant (M.Z.) and a network grant from the European Society of Pediatric Gastroenterology, Hepatology, and Nutrition (ESPGHAN).

\section{Author details \\ 'Department of Paediatrics, University of Cambridge, Addenbrooke's Hospital, Cambridge, UK. ${ }^{2}$ Department of Paediatric Gastroenterology, Hepatology and Nutrition, Cambridge University Hospitals, Addenbrooke's, Cambridge, UK. ${ }^{3}$ Wellcome Trust-Medical Research Council Stem Cell Institute, University of Cambridge, Cambridge, UK}

\section{Conflict of interest}

The authors declare no competing interests.

\section{Publisher's note}

Springer Nature remains neutral with regard to jurisdictional claims in published maps and institutional affiliations.

Received: 26 November 2020 Revised: 10 February 2021 Accepted: 3 March 2021.

Published online: 18 October 2021

\section{References}

1. Rutkowski, S. et al. Biological material collection to advance translational research and treatment of children with CNS tumours: position paper from the SIOPE Brain Tumour Group. Lancet Oncol. 19, e419-e428 (2018).

2. Lewis, C. et al. Building a 'Repository of Science': the importance of integrating biobanks within molecular pathology programmes. Eur. J. Cancer 67, 191-199 (2016).

3. Shaw, P. M. \& Patterson, S. D. The value of banked samples for oncology drug discovery and development. J. Natl Cancer Inst. - Monogr. 2011, 46-49 (2011).

4. Jackson, D. H. \& Banks, R. E. Banking of clinical samples for proteomic biomarker studies: a consideration of logistical issues with a focus on preanalytical variation. Proteom. - Clin. Appl. 4, 250-270 (2010).

5. Coppola, L. et al. Biobanking in health care: Evolution and future directions. J. Transl. Med. 17, 172 (2019).

6. Malsagova, K. et al. Biobanks-A platform for scientific and biomedical research. Diagnostics 10, 485 (2020).

7. Sachs, N. et al. A Living Biobank of Breast Cancer Organoids Captures Disease Heterogeneity. Cell 172, 373-386.e10 (2018).

8. Bolck, H. A. et al. Cancer sample biobanking at the next level: combining tissue with living cell repositories to promote precision medicine. Front. Cell Dev. Biol. 7, 246 (2019).

9. Vlachogiannis, G. et al. Patient-derived organoids model treatment response of metastatic gastrointestinal cancers. Science 359, 920-926 (2018).

10. Calandrini, C. et al. An organoid biobank for childhood kidney cancers that captures disease and tissue heterogeneity. Nat. Commun. 11, 1310 (2020).

11. Kim, J., Koo, B. K. \& Knoblich, J. A. Human organoids: model systems for human biology and medicine. Nat. Rev. Mol. Cell Biol. 21, 571-584 (2020).

12. Lancaster, M. A. et al. Cerebral organoids model human brain development and microcephaly. Nature 501, 373-379 (2013).

13. Huch, M. et al. In vitro expansion of single Lgr5 + liver stem cells induced by Wnt-driven regeneration. Nature 494, 247-250 (2013).

14. Broutier, L. et al. Culture and establishment of self-renewing human and mouse adult liver and pancreas 3D organoids and their genetic manipulation. Nat. Protoc. 11, 1724-1743 (2016).

15. Sato, T. et al. Long-term expansion of epithelial organoids from human colon, adenoma, adenocarcinoma, and Barrett's epithelium. Gastroenterology 141, 1762-1772 (2011)

16. Meran, L. et al. Engineering transplantable jejunal mucosal grafts using patientderived organoids from children with intestinal failure. Nat. Med. https://doi. org/10.1038/s41591-020-1024-z (2020).

17. Kasendra, M. et al. Development of a primary human Small Intestine-on-aChip using biopsy-derived organoids. Sci. Rep. https://doi.org/10.1038/s41598018-21201-7 (2018).

18. Sato, T. et al. Single Lgr5 stem cells build crypt-villus structures in vitro without a mesenchymal niche. Nature 459, 262-265 (2009). 
19. Yoshida, S., Miwa, H., Kawachi, T., Kume, S. \& Takahashi, K. Generation of intestinal organoids derived from human pluripotent stem cells for drug testing. Sci. Rep. 10, 1-11 (2020).

20. d'Aldebert, E. et al. Characterization of human colon organoids from inflammatory bowel disease patients. Front. Cell Dev. Biol. 8, 363 (2020).

21. Min, S., Kim, S. \& Cho, S. W. Gastrointestinal tract modeling using organoids engineered with cellular and microbiota niches. Exp. Mol. Med. 52, 227-237 (2020).

22. Takahashi, Y. et al. A refined culture system for human induced pluripotent stem cell-derived intestinal epithelial organoids. Stem Cell Rep. 10, 314-328 (2018).

23. Noguchi, T. A. K. et al. Generation of stomach tissue from mouse embryonic stem cells. Nat. Cell Biol. 17, 984-993 (2015).

24. McCracken, K. W., Howell, J. C., Wells, J. M. \& Spence, J. R. Generating human intestinal tissue from pluripotent stem cells in vitro. Nat. Protoc. 6, 1920-1928 (2011).

25. Kraiczy, J. et al. DNA methylation defines regional identity of human intestinal epithelial organoids and undergoes dynamic changes during development. Gut 68, 49-61 (2019).

26. Zachos, N. C. et al. Human Enteroids/Colonoids and Intestinal Organoids Functionally Recapitulate Normal Intestinal Physiology and Pathophysiology * (2015). https://doi.org/10.1074/jbc.R114.635995

27. Middendorp, S. et al. Adult stem cells in the small intestine are intrinsically programmed with their location-specific function. Stem Cells 32, 1083-1091 (2014).

28. Van De Wetering, M. et al. Prospective derivation of a living organoid biobank of colorectal cancer patients. Cell 161, 933-945 (2015).

29. Fujii, M. et al. A colorectal tumor organoid library demonstrates progressive loss of Niche factor requirements during tumorigenesis. Cell Stem Cell 18, 827-838 (2016)

30. Dekkers, J. F. et al. Characterizing responses to CFTR-modulating drugs using rectal organoids derived from subjects with cystic fibrosis. Sci. Transl. Med. 8, 344ra84-344ra84 (2016).

31. Sugimoto, S. et al. Reconstruction of the human colon epithelium in vivo. Cell Stem Cell 22, 171-176.e5 (2018).

32. Driehuis, E., Kretzschmar, K. \& Clevers, H. Establishment of patient-derived cancer organoids for drug-screening applications. Nat. Protoc. https://doi.org/ 10.1038/s41596-020-0379-4 (2020)

33. Seino, T. et al. Human pancreatic tumor organoids reveal loss of stem cell niche factor dependence during disease progression. Cell Stem Cell 22, 454-467.e6 (2018)

34. Tiriac, $H$. et al. Organoid profiling identifies common responders to chemotherapy in pancreatic cancer. Cancer Discov. 8, 1112-1129 (2018).

35. Yan, H. H. N. et al. A Comprehensive human gastric cancer organoid biobank captures tumor subtype heterogeneity and enables therapeutic screening. Cell Stem Cell 23, 882-897.e11 (2018).

36. Geurts, M. H. et al. CRISPR-Based Adenine Editors Correct Nonsense Mutations in a Cystic Fibrosis Organoid Biobank. Cell Stem Cell 26, 503-510. e7 (2020).

37. Driehuis, E. et al. Pancreatic cancer organoids recapitulate disease and allow personalized drug screening. Proc. Natl Acad. Sci. USA 116, 26580-26590 (2019).

38. Seidlitz, T. et al. Human gastric cancer modelling using organoids. Gut $\mathbf{6 8}$ 207-217 (2019).

39. Riordan, J. R. et al. Identification of the cystic fibrosis gene: cloning and characterization of complementary DNA. Science 245, 1066-1073 (1989).

40. Berkers, G. et al. Rectal organoids enable personalized treatment of cystic fibrosis. Cell Rep. 26, 1701-1708 (2019).

41. Schwank, G. et al. Functional repair of CFTR by CRISPR/Cas9 in intestinal stem cell organoids of cystic fibrosis patients. Cell Stem Cell 13, 653-658 (2013).

42. Pleguezuelos-Manzano, C. et al. Establishment and culture of human intestinal organoids derived from adult stem cells. Curr. Protoc. Immunol. https://doi.org/ 10.1002/cpim.106 (2020).

43. Sato, T. \& Clevers, H. Growing self-organizing mini-guts from a single intestinal stem cell: Mechanism and applications. Science https://doi.org/10.1126/ science.1234852 (2013).

44. Fujii, M., Matano, M., Nanki, K. \& Sato, T. Efficient genetic engineering of human intestinal organoids using electroporation. Nat. Protoc. 10, 1474-1485 (2015).

45. VanDussen, K. L., Sonnek, N. M. \& Stappenbeck, T. S. L-WRN conditioned medium for gastrointestinal epithelial stem cell culture shows replicable batch-to-batch activity levels across multiple research teams. Stem Cell Res. 37 101430 (2019).

46. VanDussen, K. L. et al. Development of an enhanced human gastrointestinal epithelial culture system to facilitate patient-based assays. Gut https:/doi.org/ 10.1136/gutjnl-2013-306651 (2015).

47. Miao, Y. et al. Next-generation surrogate Wnts support organoid growth and deconvolute frizzled pleiotropy in vivo. Cell Stem Cell 27, 840-851.e6 (2020).

48. Jung, P. et al. Isolation and in vitro expansion of human colonic stem cells. Nat Med. 17, 1225-1227 (2011).

49. Fujii, M. et al. Human intestinal organoids maintain self-renewal capacity and cellular diversity in Niche-inspired culture condition. Cell Stem Cell https://doi. org/10.1016/j.stem.2018.11.016 (2018).

50. Caliari, S. R. \& Burdick, J. A. A practical guide to hydrogels for cell culture. Nat. Methods 13, 405-414 (2016)

51. Hughes, C. S., Postovit, L. M. \& Lajoie, G. A. Matrigel: a complex protein mixture required for optimal growth of cell culture. Proteomics 10, 1886-1890 (2010).

52. Cruz-Acuña, R. et al. Synthetic hydrogels for human intestinal organoid generation and colonic wound repair. Nat. Cell Biol. 19, 1326-1335 (2017).

53. Aisenbrey, E. A. \& Murphy, W. L. Synthetic alternatives to Matrigel. Nat. Rev. Mater. https://doi.org/10.1038/s41578-020-0199-8 (2020).

54. Velasco, V., Shariati, S. A. \& Esfandyarpour, R. Microtechnology-based methods for organoid models. Microsyst. Nanoeng. 6, 76 (2020).

55. Zhao, Z. et al. Composite hydrogels in three-dimensional in vitro models Front. Bioeng. Biotechnol. 8, 611 (2020).

56. Coecke, S. et al. Guidance on Good Cell Culture Practice. A Report of the Second ECVAM Task Force on Good Cell Culture Practice. In Cell Technology for Cell Products (ed Smith, R.) 313-315 (Springer, Netherlands, 2007).

57. Dossena, M. et al. Standardized GMP-compliant scalable production of human pancreas organoids. Stem Cell Res. Ther. 11, 94 (2020).

58. Walsh, A. J., Cook, R. S., Sanders, M. E., Arteaga, C. L. \& Skala, M. C. Drug response in organoids generated from frozen primary tumor tissues. Sci. Rep. 6, 1-11 (2016).

59. Tsai, Y.-H. et al. RESEARCH LETTER: A method for cryogenic preservation of human biopsy specimens and subsequent organoid culture. https://doi.org/ 10.1016/j.jcmgh.2018.04.008 (2018).

60. Bui, B. N. et al. Organoids can be established reliably from cryopreserved biopsy catheter-derived endometrial tissue of infertile women. Reprod. Biomed. Online 41, 465-473 (2020).

61. Han, S. H. et al. Long-term culture-induced phenotypic difference and efficient cryopreservation of small intestinal organoids by treatment timing of Rho kinase inhibitor. World J. Gastroenterol. 23, 964-975 (2017).

62. Konnikova, L. et al. High-dimensional immune phenotyping and transcriptional analyses reveal robust recovery of viable human immune and epithelial cells from frozen gastrointestinal tissue. Mucosal Immunol. https:/doi.org/ 10.1038/s41385-018-0047-y (2018).

63. Yao, $Y$. et al. Patient-derived organoids predict chemoradiation responses of locally advanced rectal cancer. Cell Stem Cell 26, 17-26.e6 (2020).

64. Li, X. et al. Organoid cultures recapitulate esophageal adenocarcinoma heterogeneity providing a model for clonality studies and precision therapeutics. Nat. Commun. 9, 1-13 (2018).

65. De Angelis, M. L. et al. Colorectal cancer spheroid biobanks: multi-leve approaches to drug sensitivity studies. Cell Biol. Toxicol. 34, 459-469 (2018).

66. Lewis, S. K. et al. DNA methylation analysis validates organoids as a viable model for studying human intestinal aging. CMGH 9, 527-541 (2020).

67. Takai-lgarashi, T. et al. Security controls in an integrated Biobank to protect privacy in data sharing: rationale and study design. BMC Med. Inform. Decis. Mak. 17, 100 (2017)

68. Bredenoord, A. L., Clevers, H. \& Knoblich, J. A. Human tissues in a dish: The research and ethical implications of organoid technology. Science $\mathbf{3 5 5}$ 6322 (2017).

69. Mikkelsen, R. B., Gjerris, M., Waldemar, G. \& Sandøe, P. Broad consent for biobanks is best-provided it is also deep. BMC Med. Ethics 20, 71 (2019).

70. Boers, S. N., Delden, J. J., Clevers, H. \& Bredenoord, A. L. Organoid biobanking: identifying the ethics. EMBO Rep. 17, 938-941 (2016).

71. Cambon-Thomsen, A. The social and ethical issues of post-genomic human biobanks. Nat. Rev. Genet. 5, 866-873 (2004).

72. Boers, S. N., Van Delden, J. J. M. \& Bredenoord, A. L. Organoids as hybrids: Ethical implications for the exchange of human tissues. J. Med. Ethics 45, 131-139 (2019). 